
Revue
de l'histoire des religions
Revue de l'histoire des religions
$3 \mid 2009$
La culture gallicane. Références et modèles (droit, ecclésiologie, histoire)

\title{
Jacques Leschassier, Senlis et les libertés de l'Eglise gallicane (1607)
}

Jacques Leschassier, Senlis and the Freedoms of the Gallican Church (1607)

Thierry Amalou

\section{(2) OpenEdition \\ Journals}

Édition électronique

URL : http://journals.openedition.org/rhr/7277

DOI : $10.4000 /$ rhr.7277

ISSN : 2105-2573

Éditeur

Armand Colin

\section{Édition imprimée}

Date de publication : 1 juillet 2009

Pagination : 445-466

ISBN : 978-2200-92591-8

ISSN : 0035-1423

Référence électronique

Thierry Amalou, « Jacques Leschassier, Senlis et les libertés de l'Eglise gallicane (1607) », Revue de l'histoire des religions [En ligne], 3 | 2009, mis en ligne le 01 juillet 2012, consulté le 20 avril 2019. URL http://journals.openedition.org/rhr/7277 ; DOI : 10.4000/rhr.7277 


\section{Jacques Leschassier, Senlis et les libertés de l'Église gallicane (1607)}

Dans le contexte politique de l'Interdit de Venise, Jacques Leschassier, avocat au Parlement et correspondant de Paolo Sarpi, prend la défense des chanoines de Senlis. Ces derniers s'opposent à leur évêque qui, en vertu de la législation tridentine, leur refuse le droit de délivrer des lettres dimissoires. La publication de travaux érudits affirmant reconstituer le code canonique de l'Église primitive accompagne la victoire de l'avocat parisien. Le déroulement du procès et ses enjeux ecclésiologiques éclairent deux aspects de sa réception: la publicité enthousiaste qu'en firent les grands gallicans parisiens, localement, la signification des libertés gallicanes et les conséquences sociales de l'affaiblissement du pouvoir épiscopal.

\section{Jacques Leschassier, Senlis and the Freedoms of the Gallican Church (1607)}

In the political context of the Interdict of Venice, Jacques Leschassier, parliamentary lawyer and correspondent of Paolo Sarpi, defended the Canons of Senlis. They had opposed their bishop, who, in accordance with Tridentine legislation, had refused them the right to grant dimissory letters. Leschassier's victory was accompanied by the publication of scholarly works claiming to reconstitute the canon code of the primitive Church. The way the trial played out and its ecclesiological stakes help to illuminate two aspects of its reception: the enthusiastic publicity that the major Paris Gallicans made of it and, locally, the meaning of Gallican freedoms and the social consequences of the weakening of episcopal power. 
Comment un événement local presque insignifiant dans son apparente banalité - un conflit de juridiction entre le chapitre cathédral de Senlis et son évêque - devint-il en 1607 un procès retentissant capable de mettre en émoi les grands érudits gallicans de la capitale sensibles à la publicité qu'en fit l'avocat au Parlement Jacques Leschassier?

Selon Jacques-Auguste de Thou, lorsque Leschassier prit la défense du chapitre cathédral de Senlis, qui prétendait pouvoir accorder des lettres dimissoires à ses membres désirant être ordonnés dans un autre diocèse que celui de Senlis, il publia des pièces de procédure constituant «des monuments en faveur des libertés de l'Eglise gallicane $»^{1}$. Pierre de L'Estoile adopte un jugement semblable en fixant son attention sur les «lettres d'appel comme d'abus ", réunies sous forme de factums, que lui avait transmises son ami Pierre Dupuy. L'Estoile gardait d'ailleurs précieusement ces documents « notables pour la défense des libertés gallicanes $»^{2}$ dans l'un de ses manuscrits in-folio.

Quels étaient donc les ressorts de cette affaire qui se déroule dans un contexte d'effervescence politique suscitée par les répercussions en France de l'Interdit de Venise? Les gallicans parisiens, dont Leschassier, solidaires de la Sérénissime et de Paolo Sarpi, entendaient s'opposer publiquement aux prétentions jugées inacceptables du pape Paul V. Qu'entendait-on par "libertés gallicanes », alors que Leschassier venait précisément de publier en 1606 un court traité sur La liberté ancienne et canonique de l'Eglise gallicane adressé aux cours souveraines ${ }^{3}$ et qu'un an plus tard était élaboré un projet de concile gallican désireux d'affirmer le statut bien particulier de l'Église de France ${ }^{4}$ ?

Afin de mieux saisir l'articulation entre les positions ecclésiologiques des chanoines de Senlis et le gallicanisme royal ou parlep. 229.

1. Jacques-Auguste de Thou, Histoire universelle, La Haye, t. X, 1740,

2. Mémoires-journaux de Pierre de L'Estoile, éd. Gustave Brunet et alii (1875), rééd. Paris, 1982, t. IX, p. 19.

3. Jacques Leschassier, De la liberté ancienne et canonique de l'Eglise gallicane aux cours souveraines de France, Paris, chez Claude Morel,1606.

4. Corrado Vivanti, Lotta politica e pace religiosa in Francia fra Cinque et Seicento, Turin, 1963. 
mentaire de leurs défenseurs ${ }^{5}$, nous examinerons la configuration sociale de Senlis, ville de catholiques royaux qui tiraient fierté de leur fidélité aux souverains légitimes pendant la Ligue. L'analyse des enjeux canoniques et juridiques des thèses de Leschassier nous aidera ensuite à mieux en apprécier la réception locale.

\section{LeS ORIGINES de L'AFFAIRE : SENLIS, UN CHAMP d'ACTION IDÉAL POUR LE GALLICANISME PARLEMENTAIRE}

Le choix de Senlis n'est sans doute pas fortuit dans la carrière de Jacques Leschassier. Ce dernier s' y était réfugié pendant la Ligue partageant alors le destin d'hommes qui après avoir été fidèles à Henri III s'étaient ralliés sans état d'âme à Henri IV. La notoriété de l'avocat parisien ne tenait pas seulement à sa réputation d'érudit mais également à une volonté farouche de servir, au barreau ou au parquet, les souverains légitimes et l'État monarchique.

\section{Pourquoi Jacques Leschassier? La linéarité d'une vie d'érudition au service du pouvoir royal}

De fait, ce fils d'un secrétaire du roi ${ }^{6}$, né à Paris en 1550 , dut à la faveur d'Henri III et à la recommandation de Guy Du Faur de Pibrac, d'occuper les fonctions de substitut du procureur général du même Parlement ${ }^{7}$. La figure intellectuelle de Pibrac exerçait

5. Marc Venard, « Le projet d'un nouveau concile dans la France d'Henri IV », Autour du concile de Trente, Saint-Etienne, 2006, p. 51. Alain Tallon, Conscience nationale et sentiment religieux en France au XVI siècle. Essai sur la vision gallicane du monde, Paris, PUF, 2002, p. 137-138, montre qu'au-delà des divergences entre les différents types de gallicanisme (royal, parlementaire, ecclésiastique), existe un « socle commun ». Outre la tradition conciliaire, se dégage l'idée d'une «Église universelle... composée d'Églises particulières en communion les unes avec les autres sous la puissance spirituelle (et non temporelle) du pape ».

6. Pierre-Philippe Leschassier, écuyer, conseiller et secrétaire du roi avait épousé Claude Miette, fille de Jean seigneur de Bois-Raoul près d'Amiens, voir François Aubert de la Chesnaye-Desbois, Dictionnaire de la noblesse, contenant les généalogies, l'histoire et la chronologie des familles nobles de France, tome 6, Paris, rééd. 1980 , p. 893.

7. Les Cuvres de M. Jacques Leschassier, parisien, avocat en Parlement, contenant plusieurs excellents traittez tant du droit public des Romains que celuy des François. Ensemble quelques mémoires servans à l'antiquité de l'Église et à 
alors un rayonnement certain tant sur la cour (il aurait favorisé la création de l'académie du palais en 1576) que sur le monde savant de la magistrature ${ }^{8}$. Et c'est bien dans le milieu humaniste issu de l'école de droit français que Leschassier tisse alors, dans le courant des années 1570, des relations d'amitié qu'entretiennent peut-être des alliances familiales ${ }^{9}$ avec des gallicans tels que Pierre Pithou ou Antoine Loisel ${ }^{10}$.

Proche du courant des Politiques, Leschassier connaît ensuite l'exil des parlementaires qui refusèrent la Ligue et séjourne successivement à Châlons-en-Champagne et Senlis ${ }^{11}$. Le retour de la paix le voit reprendre une simple charge d'avocat au Parlement, tandis que ses complices Loisel et Pithou étaient récompensés dès 1594, devenant respectivement avocat du roi et procureur du roi au Parlement de Paris ${ }^{12}$. Tout en se consacrant au barreau, Leschassier incarne la voix du Parlement. Ainsi dès avant l'instauration de la Paulette, il aurait suggéré à Henri IV de rendre les offices héréditaires et patrimoniaux ${ }^{13}$. Il conseille également au roi de renoncer au projet de réduction des rentes sur l'hôtel de ville défendu par Sully en $1605^{14}$.

La renommée de Leschassier allait croître lors de l'affaire de l'Interdit vénitien. Avec l'avocat général du roi Louis Servin, il est

l'illustration de l'Histoire de France, Paris, 1649, p. aii-aiii pour la biographie de l'auteur composée par son neveu Christophe.

8. Jacqueline Boucher, La cour de Henri III, Rennes, 1986, p. 139; Mark Greengrass, Governing Passions. Peace and Reform in the French Kingdom, 1576-1585, Oxford, 2007, p. 46. Leschassier fit partie de l'ambassade de Pibrac en Pologne.

9. Nous ignorons malheureusement le degré de parenté entre Jacques Leschassier et Jean Leschassier, conseiller au Châtelet qui épouse Marie, fille de Pierre Pithou (Dictionnaire de la noblesse..., t. 6, p. 892).

10. Sur le mos gallicus et l'école de droit français, voir Donald Kelley, Foundations of Modern Historical Scholarship : Language, Law, and history in the French Renaissance, New-York et Londres, 1970.

11. Les Euvres de M. Jacques Leschassier..., pièces liminaires : Elogium Iacobi Leschassieri IC parisensis.

12. D. Kelley, op. cit., p. 256.

13. Le discours serait contemporain de l'assemblée des notables de 1596, voir Roland Mousnier, La Vénalité des offices sous Henri IV et Louis XIII, Paris, 1971, p. 558.

14. BnF, coll. Dupuy, ms $240, \mathrm{n}^{\circ} 150$ : « remontrance de Leschassier au roi sur les rentes », éditée dans Les Euvres... (p. 243) avec la remontrance du prévôt des marchands François Miron sur le même sujet. 
en effet l'un des deux juristes français ${ }^{15}$ à soutenir publiquement la République de Venise en rédigeant une « défense » face aux prétentions du pape Paul V. Reconnaissante, la Sérénissime lui fit parvenir une chaîne d'or en récompense ${ }^{16}$. À cette occasion, l'ambassadeur de Venise à Paris, Pietro Priuli, joua un grand rôle dans l'orchestration de la controverse éditoriale en multipliant les contacts entre les adversaires de Rome ${ }^{17}$. Outre Servin et Leschassier, on retrouve dans cette « internationale gallicane et antiromaine » des hommes issus d'horizons divers : le protestant Isaac Casaubon ou encore le théologien de Sorbonne Edmond Richer qui soutint l'entreprise éditoriale de réédition de l'œuvre de Gerson pour faire pièce aux prétentions pontificales ${ }^{18}$. Tous s'inquiétaient des progrès d'une doctrine pontificale fondée sur la plenitudo potestatis et relayée par une ecclésiologie tridentine peu soucieuse des particularismes locaux ou nationaux ${ }^{19}$.

Pierre de L'Estoile rend bien compte de l'intérêt suscité alors par la polémique liée à l'Interdit de Venise et de l'estime qu'il porte, pour son originalité, au libelle de Leschassier: "Bérion le même jour (jeudi 21 décembre 1506) m'a donné ung discours latin de M. Leschassier sur le différend du pape et des Vénitiens qu'il venoit d'achever d'imprimer et tirer de dessous la presse : il est intitulé Consultatio parisii cujusdam de controversia inter sanctitatatem Pauli V et Sereniss. Rempublicam venetam [ad virum clariss. Venetum]. Ce discours est bien fait et à mon jugement n'en doit rien aux autres $»^{20}$.

15. Sylvio Hermann De Franceschi, «La genèse française du catholicisme d'État et son aboutissement au début du ministériat de Richelieu. Les catholiques zélés à l'épreuve de l'affaire Santarelli et la clôture de la controverse autour du pouvoir pontifical au temporel », Annuaire bulletin de l'histoire de France (année 2001), 2003, p. 24-25.

16. Mémoires-journaux de Pierre de L'Estoile, t. 8, p. 258 (nov. 1606).

17. Pietro Priuli souhaitait rassembler les écrits français parlant « de l'excommunication, de l'autorité du pape et de ses entreprises », voir C. Vivanti, Guerre civile et paix religieuse dans la France d'Henri IV, Paris, 2006, p. 164-165.

18. Philippe Denis, «Edmond Richer, protestant malgré lui? », in De l'humanisme aux Lumières, Bayle et le protestantisme. Mélanges en l'honneur d'Elisabeth Labrousse, Paris-Oxford, 1996, p. 343-358. Dès 13 septembre 1606, Richer avait promis à Priuli d'écrire en faveur de Venise : Gaetano Cozzi, Paolo Sarpi tra Venezia e l'Europa, Turin, 1979, p. 74 n. 25.

19. William J. Bouwsma, "Gallicanism and the Nature of Christendom », in Renaissance Studies in honor of Hans Baron, éd. A. Molho et J. Tedeschi, Dekalb (Ill.), Northern Illinois University Press, 1971, p. 308-324.

20. Mémoires-journaux de Pierre de L'Estoile, t. 8, p. 261-262. 
Fidèle aux usages des humanistes, Leschassier débute alors en septembre 1607 une correspondance suivie avec Paolo Sarpi ${ }^{21}$. Il est possible qu'Isaac Casaubon ou Jérôme Groslot de l'Isle soient à l'origine de cette rencontre épistolaire qui se poursuit jusqu'en $1613^{22}$. Enfin, la célébrité de Leschassier dans le cercle des gallicans du Parlement de Paris tenait à ses travaux de canoniste : la publication De la liberté ancienne et canonique de l'Église gallicane en 1606 venait accréditer l'idée que les apôtres avaient légué un code canonique contenant les « libertés anciennes » de l'Église primitive. L'examen des quatre premiers conciles œcuméniques révélait les traces de cette législation jusque-là méconnue ${ }^{23}$. Le développement du droit canon, particulièrement après le décret de Gratien, aurait réduit cette «vraie, naturelle et absolue liberté de l'Eglise $»^{24}$. Fort heureusement, l'autorité des rois de France depuis saint Louis aurait permis de maintenir des « libertés récentes » sans désobéir pour autant au Saint-Siège.

C'est donc un juriste et canoniste de réputation internationale qui prend à bras le corps l'affaire des chanoines de Senlis. De même qu'il avait combattu la tyrannie pontificale en défendant Venise, Leschassier pouvait espérer trouver une illustration concrète de ses théories sur les libertés anciennes et récentes de l'Église gallicane. La proximité de Paris d'une ville où son ami Antoine Loisel conservait plusieurs parents ne pouvait que l'y encourager.

21. BnF, coll. Dupuy, ms 251 : copie de Pierre Dupuy des lettres latines de Sarpi à Leschassier du 7 septembre 1607 au 23 juillet 1613; voir Boris Ulianich, Lettere ai gallicani-Paolo Sarpi, Wiesbaden, 1961. Sur le protestant Jérome II Groslot de l'Isle, voir Eugène et Émile Haag, La France protestante, Paris, 18461859 , rééd. Genève, 1966, t. 5, p. 371.

22. Gaetano Cozzi, Paolo Sarpi tra Venezia e l'Europa, Turin, 1979, p. 115. En effet, tous les nouveaux correspondants français de Sarpi en 1607 sont aussi des amis d'Isaac Casaubon. BnF, ms Dupuy 251 : copie de Pierre Dupuy des lettres latines de Sarpi à Leschassier du 7 septembre 1607 au 23 juillet 1613.

23. Jacques Leschassier, De la liberté ancienne et canonique..., p. 29.

24. Joseph Leclerc, "Qu'est-ce que les libertés de l'Église gallicane?», Recherches de science religieuse, XXIII, 1933, p. 556, montre que Leschassier s'appuie sur le huitième canon d'Éphèse qui s'opposait aux prétentions de l'évêque d'Antioche à ordonner les évêques de Chypre. Le retranchement de ce canon à la législation canonique ultérieure relèverait d'une « conspiration romaine ». Le travail de restitutio de Leschassier consiste alors à transférer le principe de ce canon (et du code primitif auquel il se rattache) aux relations de l'Église gallicane avec le pape. 


\section{Une ville royale opposée à l'intransigeance d'un jeune évêque tridentin}

Quels sont les traits dominants de Senlis qui incarne si bien le catholicisme royal au début du XVII ${ }^{\mathrm{e}}$ siècle? La permanence historique de la fidélité monarchique, exposée sous forme d'emblème, s'impose comme un mythe urbain forgé par l'expérience de la Ligue. Ainsi Nicolas Barthélemy, avocat au Parlement, dont le père fut l'un des plus fidèles soutiens d'Henri IV ${ }^{25}$, se glorifie d'être « habitant d'une ville inviolablement attachée au service de ses roys et dont la gloire luy a merité cette devise, Qu'elle n'a jamais souillé de la moindre tâche d'infidélité la blancheur de ses lys $»^{26}$.

De fait, les quelques ligueurs extrémistes, au premier rang desquels figurait l'évêque Guillaume Rose, ne parvinrent pas à s'imposer au sein des notabilités, et la ville chassa les plus intransigeants d'entre eux après le 25 avril 1589. Dominée par les officiers royaux, l'oligarchie municipale vante une forme de tempérance, d'action raisonnable (fondée notamment sur un fort attachement à l'État monarchique), sans se départir pour autant de son désir d'unité religieuse au sein d'une Église catholique restaurée ${ }^{27}$. La double fidélité de Senlis aux souverains légitimes et au catholicisme distingue ce bastion royaliste d'où Henri IV, peu après son sacre de Chartres, devait partir pour recevoir la soumission des Parisiens ${ }^{28}$.

Privée de son évêque banni jusqu'en 1596, Senlis développe un système festif et commémoratif puissant. Les Te Deum et les processions-messes extraordinaires rythment les succès militaires du roi en confortant une vision providentialiste de l'histoire; celleci atteste de l'élection divine de la ville aux côtés d'Henri IV, tandis

25. Noël Valois, Inventaire des arrêts du Conseil d'État (règne de Henri IV), Paris, Imprimerie nationale, 1886, t. I, p. 69 n 1120 : Nicolas I Barthélemy, procureur du roi au bailliage de Senlis obtient une rente de 200 écus jusqu'à remboursement de la somme avancée par lui au roi (14 juillet 1594).

26. Nicolas Barthélemy, Apologie du banquet sanctifié de la fête des rois, Paris, 1664, épître dédicatoire.

27. Thierry Amalou, Le lys et la mitre. Loyalisme monarchique et pouvoir épiscopal à Senlis pendant la Ligue (vers 1580-vers 1610), Paris, Éditions du Comité des Travaux Historiques et Scientifiques, 2007.

28. C'est la déclaration de Senlis du 20 mars 1594 dans laquelle le roi exprime sa clémence et sa volonté de réunir tous ses sujets dans la paix : le texte de cette déclaration fut distribué par les troupes royales lors de l'entrée d'Henri IV à Paris le 22 mars 1594, voir à ce sujet Maïthé Vallès-Bled (dir.), 1594 Le sacre d'Henri IV à Chartres, Chartres, 1994, p. 266. 
qu'un véritable culte monarchique fusionne habilement avec les traditions locales. Or ces dernières, notamment les dévotions rendues à Rieul (le saint patron évangélisateur de la ville) étaient porteuses de consensus social. De surcroît, elles avaient fait l'objet d'une restauration gallicane menée en continuité par les évêques depuis les années $1520^{29}$.

Dans cette société urbaine où règnent les officiers moyens du présidial, rares sont ceux qui se rapprochent des robins parisiens. Seuls les Loisel sortent un peu de la médiocrité des offices subalternes : Claude Loisel, lieutenant général du bailliage de Senlis, et neveu du célèbre jurisconsulte, devient Président de la cour des aides en $1608^{30}$. Il est tentant d'imaginer que c'est par son truchement que Leschassier, ami de son oncle Antoine Loisel, entra en contact avec les chanoines de Senlis. De surcroît parmi les dignitaires du chapitre figurait l'archidiacre Jean-Philippe Loisel, autre neveu de l'avocat parisien $^{31}$.

Enfin, Senlis est une ville dont le nouvel évêque Antoine Rose, neveu du précédent, s'est rapidement aliéné les notables, la municipalité ainsi qu'une partie du clergé. Lors de l'entrée épiscopale du 24 mars 1602 - parcours symbolique et codifié à l'occasion duquel le prélat prêtait serment de respecter les privilèges des différents corps constitués tout en honorant saint Rieul ${ }^{32}$ - Antoine Rose refusa de suivre à la lettre le cérémonial établi ${ }^{33}$. Ainsi, il s'abstint de l'offrande d'un drap de soie à l'autel de la collégiale Saint-Rieul,

29. Thierry Amalou, Une concorde urbaine. Senlis au temps des réformes (1520-1580), Limoges, Presses universitaires de Limoges, 2007, p. 177-270.

30. Scourion de Beaufort, «Les Loisel, une famille de lieutenants généraux du bailliage de Senlis, $\mathrm{XVI}^{\mathrm{e}}-\mathrm{XV} I I^{\mathrm{e}}$ siècles ", Mém. Soc. hist. arch. de Senlis, t. III, 1899 , p. 24.

31. Jean-Philippe Loisel devient archidiacre le 10 mai 1602 : Arch. dép. Oise, G 2022.

32. Antoine Rose, docteur en droit canon, fils de Nicolas Rose secrétaire de la chambre du roi et neveu du précédent évêque Guillaume Rose, n'était âgé que de 26 ans lorsqu'il reçoit ses bulles de provision (12 novembre 1601). Il permute son siège avec celui de Clermont alors occupé par François de La Rochefoucauld le $1^{\text {er }}$ mars 1610. Voir Joseph Bergin, The Making of the French Episcopate 1589-1661, New Haven et Londres, 1996, p. 692. Id., Cardinal de La Rochefoucauld. Leadership and Reform in the French Church, New Haven et Londres, 1987, p. 92-118.

33. Arch. dép. Oise, BB 6, fol. $511 \mathrm{v}^{0}:$ « C'est la manière de l'entrée du nouvel evesque de Senlis et ce qu'il doibt faire en son joieulx advenement $»$. Ibid., fol. $512 \mathrm{v}^{\mathrm{o}}$ : «C'est ce que doibt jurer ledict evesque aux gouverneurs et habitans de ladicte ville $»$. 
rituel qui selon la Tradition visait à honorer le premier évêque et saint patron de la ville. C'est l'origine d'un conflit qui donna lieu à un long procès marqué par une première décision du Parlement, en décembre 1609, condamnant Rose à payer le drap d'or ${ }^{34}$.

En outre, une partie du clergé soutenue par l'évêque, avait cru bon de protester contre les commémorations du siège de 1589 considérées comme des corvées ${ }^{35}$. Le jeune évêque, typique de cette génération de prélats désireux d'imiter Charles Borromée en sanctifiant la vie quotidienne et en renforçant la frontière entre la liturgie sacrée des clercs et les activités profanes des laïcs, voyait sans doute d'un mauvais œil ces commémorations récentes imposées au calendrier liturgique par les royaux. D'autant que ces dernières rappelaient la défaite des ligueurs que son oncle avait soutenus ${ }^{36}$.

Mais le refus de participer à des cérémonies désormais inscrites au cœur de l'identité urbaine fut ressenti que comme une inacceptable provocation par le corps de ville. La municipalité relaya le chapitre Saint-Rieul dans les récriminations anti-épiscopales. La situation s'envenima au point qu'Antoine Rose en vint à proférer des injures à l'encontre des échevins; ceux-ci portèrent alors l'affaire devant le Parlement qui leur donna raison ${ }^{37}$. Cette décision était la première d'une série de jugements qui allaient isoler Rose non seulement de l'élite dirigeante, scandalisée de la remise en cause du culte du saint patron et du système commémoratif issu de la Ligue, mais également des principaux collèges de chanoines. C'est dans ce contexte local d'unanimisme anti-épiscopal que se déroule « l'affaire des chanoines de Senlis ».

34. Arch. dép. Oise : G 2720 (dossier 9-10). Le procès entre Rose et les chanoines de la collégiale Saint-Rieul se poursuivit au moins jusqu'en 1639, bien après le départ de Rose (1610).

35. Jean Vaultier, Histoires et discours d'une partie des choses faites et passées en ce royaume qui ont eu cours depuis le 13 mai 1588 jusqu'au 16 juin 1598 , éd. A. Bernier in Monuments inédits de l'histoire de France, Paris, 1834, p. 211. Le 6 juillet 1603 la ville confirme l'inscription de la commémoration, Arch. dép. Oise, BB 6, fol. $517 \mathrm{r}^{\circ}$.

36. Correspondance du nonce en France Innocenzo del Bufalo, évêque de Camerino, éd. Bernard Barbiche, Rome-Paris, (« Acta Nuntiaturae Gallicae » 4), 1964, p. $192 \mathrm{n}^{\circ} 41$ : le nonce, conscient des risques de conflits locaux que ne manquerait pas de susciter l'arrivée de ce jeune et impétueux neveu de Guillaume Rose, tenta en vain de retarder l'expédition des bulles de provision.

37. Arch. dép. Oise, BB 6 , fol. $561 \mathrm{r}^{\circ}-\mathrm{v}^{\circ}$ : reproduit un extrait des registres du Parlement du 6 juillet 1603. 


\section{ENJEUX JURIDIQUES ET ECCLÉSIOLOGIQUES : LES PRIVILÈGES DU CHAPITRE CATHÉDRAL COMME VESTIGES D'UN CODE CANONIQUE DE L'ÉGLISE PRIMITIVE}

Au nom du gallicanisme ecclésiastique les chanoines entendaient défendre un ensemble de coutumes "anciennes et mémorables », antérieures au concile de Trente, auxquelles le droit canon de l'Église s'était adapté. Ainsi, forte des privilèges juridictionnels anciens accordés par l'évêque Henri à la fin du XII ${ }^{\mathrm{e}}$ siècle $^{38}$, l'officialité du chapitre veillait à la dignité de la célébration des messes des défunts en exigeant que les prêtres habitués et chapelains soient ordonnés prêtres ${ }^{39}$. En 1608 encore, une ordonnance capitulaire enjoignait à François Fortier, chapelain des obits, de se faire rapidement conférer l'ordre de prêtrise sous peine d'en pourvoir un autre plus digne à sa place ${ }^{40}$.

En souhaitant interdire au chapitre le droit d'accorder des lettres dimissoires, pratique qui s'était probablement développée lors de la vacance du siège épiscopal entre 1589 et $1596^{41}$, Rose revenait sur le serment de respecter les privilèges du chapitre, serment prêté lors de son entrée solennelle. Les chanoines portèrent l'affaire devant le Conseil d'État dès $1604^{42}$. Pourtant, l'évêque ne faisait que reprendre les dispositions statutaires du concile provincial de Reims de 1583 qui s'inspiraient elles-mêmes du concile de Trente ${ }^{43}$. Rose se conformait ainsi à l'épiscopalisme de la législation tridentine qui réservait aux seuls évêques le pouvoir d'ordonner et de

38. Arch. dép. Oise, G 2006; Marie-Antoinette Menier, « Le chapitre cathédral de Senlis de 1139 à $1516 »$, Soc. hist. arch. de Senlis, 1971, p. 40-42.

39. Voir à ce sujet Philippe Loupès, Chapitres et chanoines de Guyenne au XVII siècle, Paris, 1985, p. 177-178 qui décrit le monde des prêtres habitués et des chapelains comme celui d'une plèbe cléricale, souvent négligente, victime de la médiocrité des revenus des chapellenies.

40. Arch. dép. Oise, G 2730, 26 février 1608.

41. Arch. dép. Oise, G 2023 : il faut un arrêt du Parlement, le 2 août 1595, pour autoriser Arnaud Sorbin évêque de Nevers à délivrer l'acte de réception à la prêtrise de Barthélemy-Jean Thureau qui n'avait pu obtenir de lettres dimissoires du chapitre cathédral de Senlis pour devenir l'un des chapelains des obits de la cathédrale.

42. N. Valois, op. cit., t. II, $178 \mathrm{n}^{\circ} 8329$ (10 juillet 1604).

43. Le premier concile provincial tenu a Rheims, l'an 1583 par monseigneur l'illustrissime et reverendissime cardinal de Guyse, archevesque duc de Rheims... le tout corrigé par nostre saint pere le Pape Grégoire XIII et mis en françois par M. H. Meurier doyen et chanoine theologal de Reims, éd. Jean de Foigny, Reims, 1586, p. 44 : «Que nul ne demande ou obtienne lettres dimissoires quand il pourra etre ordonné par son evesque : afin qu'il ne semble avoir décliné l'examen et iugement de son evesque, pour plus aisement estre admis par un autre ». 
confirmer ${ }^{44}$. Sûr de son bon droit, Rose excommunia en 1605 le chanoine Hercule Dufresnoy, membre d'une famille influente de notables de Senlis ${ }^{45}$, précisément pour le motif que celui-ci venait de recevoir des lettres dimissoires du chapitre ${ }^{46}$. Dans le même temps, le Conseil d'État renvoyait l'affaire devant le Grand Consei ${ }^{47}$.

C'est le début d'un affrontement juridique dont la dynamique se nourrit d'un combat idéologique livré par l'impression des pièces de procédures et par l'élaboration de mémoires touchant à l'interprétation canonique des faits. Loin de se fixer sur les particularismes locaux, Leschassier entendait faire triompher publiquement l'idée d'une Église gallicane disposant non pas de privilèges mais de franchises inscrites dans un ancien code canonique remontant aux temps apostoliques ${ }^{48}$.

\section{Libelles, traités et faux en écriture : ecclésiologie gallicane versus épiscopalisme}

Une première requête soumise au Grand Conseil donnait immédiatement une portée générale à la cause défendue en assimilant les libertés du chapitre cathédral de Senlis à celles contenues dans les libertés de l'Église gallicane : «La liberté de l'église gallicane est le droit commun ancien dans lequel les chapitres des églises cathedrales trouvent la leur comme les autres estats de l'Eglise $»^{49}$. Leschassier s'efforçait en effet de démontrer que durant les premiers temps du christianisme le pouvoir d'ordination était partagé par les collèges de prêtres, ancêtres des collèges de chanoines :

« SaintPaul demeurant avec plusieurs prêtres avait ordonné Timothée auquel tous les prêtres avoient imposé les mains avec l'apôtre que les

44. Jean Bernhard, Charles Lefebvre, Francis Rapp, Histoire du droit et des institutions de l'Église en occident, $\mathrm{t}$. XIV : L'époque de la Réforme et du concile de Trente, Paris, 1990, p. 207.

45. Le lys et la mitre..., p. 334-336.

46. Arch. dép. Oise à Beauvais G 2013 : sentence d'excommunication du 7 février 1605.

47. N. Valois, op. cit., t. II, p. 251 (19 juillet 1605).

48. Leschassier ne met pas en avant les privilèges accordés par l'évêque Henri au XII ${ }^{\mathrm{e}}$ siècle et affirme que les chanoines étaient " en possession immémoriale » d'accorder des lettres dimissoires, Antoine Fontanon, Les édits et ordonnances des rois de France traittans de la police sacrée et discipline ecclésiastique, t. IV, Paris, 1611, p. 1288.

49. Les Euvres de M. Jacques Leschassier..., p. 335. 
conciles de Carthage, d'Antioche et les anciens conciles n'avoient point connu d'autre discipline [...] que c'estoit aussi le sentiment de saint Jérôme $[. .$.$] mais qu'ensuite pour contenir dans les bornes cette espèce$ de société leonine il avoit fallu partager la juridiction commune $\aleph^{50}$.

Cette argumentation reposait sur la thèse déjà évoquée de 1'existence d'un code canonique primitif, creuset des libertés gallicanes. De fait, Leschassier fournit au Grand Conseil un exemplaire de son livre sur La liberté ancienne et canonique de l'Eglise gallicane ${ }^{51}$. En se fondant sur l'examen des décisions du concile d'Éphèse, l'avocat des chanoines de Senlis s'émerveillait de ce que ce code primitif sauvegardait les particularités des Églises locales (ou nationales) : « Cette définition de la liberté n'est point particulière aux François, ils l'ont prise dans l'article 6 du concile d'Ephese, le troisième des quatre conciles œcuméniques, lequel article il ordonne expressement estre envoyé par toutes les provinces de la chrestienté $\rangle^{52}$. Fort habilement, Leschassier raccordait ensuite son œuvre de généalogie canonique et conciliaire aux travaux des parlementaires gallicans qui s'étaient efforcés, notamment pendant la Ligue, d'inventorier les libertés gallicanes pour mieux borner l'autorité pontificale ${ }^{53}$.

Opérant la jonction entre le gallicanisme ecclésiastique et le gallicanisme parlementaire, il pouvait dénoncer « l'orgueil des pasteurs de l'Eglise qui abusent de leur pouvoir sous le ministère des choses sacrées $\rangle^{54}$ en rappelant que le gallicanisme du Parlement tirait lui aussi son autorité des anciens conciles ${ }^{55}$. À la manière de Pierre Pithou $^{56}$, les thèses de Leschassier pouvaient se résumer sous forme

50. De Thou, Histoire universelle, éd. 1740, La Haye, t. X, p. 229; Antoine Fontanon, op. cit., p. 1289.

51. Les Euvres de M. Jacques Leschassier..., p. 331.

52. Les Euvres de M. Jacques Leschassier..., p. 335.

53. Les Euvres de M. Jacques Leschassier..., p. 336 : «ainsi que le récite le livre des libertez d'icelle (Eglise gallicane) publié en 1594 par l'authorité de la cour ». De la liberté ancienne et canonique..., p. 29 : «Que ni le pape ni tout le clergé ensemble ne peuvent disposer d'aucune chose temporelle » et au plan spirituel «Que tout prelat mesme le pape est suject aux conciles».

54. Les Euvres de M. Jacques Leschassier..., p. 335.

55. De la liberté ancienne et canonique..., p. 29: «Le parlement de Paris a tousjours constamment tenu par tradition ancienne escrite dans la memoire des hommes sages et experimentez que la principale liberté de l'Eglise est dans le droit des quatre premiers conciles oecumeniques $»$.

56. Pierre Pithou, Les libertés de l'Église gallicane, Paris, 1594, p. 5 : à côté d'un catalogue des Libertés de l'Eglise gallicane, la définition de ces dernières se résume en deux maximes : «La premiere, que les papes ne peuvent rien comman- 
de maxime : les « conciles modernes qui contiennent la servitude (doivent) céder aux anciens qui contiennent la liberté puisqu'elle est un droit commun, canonique, ancien $»^{57}$.

Leschassier entendait donc démontrer que les évolutions ultérieures de la législation canonique avaient occulté la juridiction, commune à l'origine, des communautés de prêtres, ancêtres des collèges de chanoines, et des évêques. Par là même, il prenait implicitement le contre-pied des réformes disciplinaires et ecclésiologiques introduites par le concile de Trente en considérant que le chapitre

« était autrefois le conseil de l'évêque, indépendant, avec une juridiction à part, aussi ancienne que celle de l'évêque. Ainsi les chanoines de Senlis ne faisoient qu'user de leurs droits, en donnant, même pendant que le siège etoit rempli, des dimissoires à leurs collègues, comme ils avoient droit d'en donner à tous les clercs sans distinction pendant la vacance $»^{58}$.

Le ton combatif et volontiers antiromain de la requête de Leschassier ne pouvait que lui attirer la sympathie des officiers de la cour souveraine. Les magistrats rendirent ainsi un arrêt favorable à la cause des chanoines le 18 mars $1606^{59}$. La lecture des pièces de procédure et du traité de Leschassier provoquèrent la colère d'Antoine Rose, non moins borroméo-tridentin et « romain » que son oncle Guillaume. Le jeune évêque tenta alors de faire censurer les textes de Leschassier dont le contenu lui semblait non seulement fallacieux mais hérétique.

\section{Un attentat contre l'honneur d'une cour souveraine...}

Rose s'adressa au chancelier Pomponne de Bellièvre, qui avait en charge la surveillance des impressions de livres ${ }^{60}$ et à Nicolas

der ny ordonner, soit en general ou en particulier de ce qui concerne les choses temporelles es pays et terres de l'obéissance et souveraineté du roy tres chrestien [...] La seconde, qu'encores que le pape soit recogneu pour souverain es choses spirituelles; toutesfois en France la puissance absolue et infinie n'a point de lieu mais est retenue et bornée par les canons et regles des anciens conciles de l'Eglise receux en ce royaume ».

57. De La Liberté ancienne et canonique..., p. 30.

58. De Thou, Histoire universelle, 1740, La Haye, t. X, p. 229.

59. Les Euvres de M. Jacques Leschassier..., p. 318.

60. Voir Olivier Poncet, Pomponne de Bellievre (1529-1607). Un homme d'État au temps des guerres de Religion, Paris, 1998, p. 242-251 qui insiste sur la 
Brulart seigneur de Sillery, garde des sceaux, qui devait succéder peu après à Bellièvre. L'évêque de Senlis demandait notamment que les écrits de Leschassier soient examinés par la Sorbonne. Débouté de sa demande ${ }^{61}$, Rose porta alors l'affaire devant l'assemblée du clergé de France créant par là même deux occasions de scandale : d'une part, il fit résumer de façon controuvée les thèses de son adversaire dans un libelle anonyme ("plein de sales et ordes paroles» nous dit Leschassier ${ }^{62}$ ); d'autre part, il soumit à la censure des propositions déjà examinées par une juridiction royale, propositions que Rose considérait « contraire(s) au droit divin et à l'ancienne discipline et (les) taxoit de fausseté, d'hérésie et d'impiété ${ }^{63}$.

Plus précisément, Rose reprochait à Leschassier plusieurs propositions qui outre leur tonalité anti-épiscopale - les conciles de Carthage et d'Ephèse auraient été «corrompus par l'ambition et l'avarice des évesques »- tendaient à attribuer aux prêtres une puissance presque égale à celle des évêques en matière d'ordination ${ }^{64}$. Au prix d'un anachronisme, on ne peut que souligner la similitude de ces propositions avec le presbytérianisme du De ecclesiastica et politica potestate d'Edmond Richer en $1611^{65}$. La comparaison peut d'ailleurs être prolongée : comme Richer, Leschassier insistait sur l'idée d'un gouvernement collégial de l'Église tant au niveau des Églises locales où les chanoines constituent le conseil de l'évêque qu'à celui de la chrétienté où le pape doit composer avec l'autorité des conciles œcuméniques ${ }^{66}$.

Jugées « faulses, erronées, heretiques, contraires au droit divin, decrets et constitutions canoniques et ressentans impiété », les thèses de Leschassier furent censurées par l'assemblée du clergé le

modération ou la passivité du chancelier en matière de censure, faute de personnel suffisant et spécialisé.

61. Procédures contre un escrit fait a l'occasion de l'ancienne et canonique liberté de l'Eglise gallicane, in Les Cuvres de M. Jacques Leschassier..., p. 318.

62. « orde », dont « ordure » est dérivée, désigne une saleté repoussante.

63. Ibid.

64. Acte du libelle diffamatoire, in Les Euvres de M. Jacques Leschassier..., p. 337.

65. Sur Richer voir Edmond Préclin, «Edmond Richer (1559-1631). Sa vie, son œuvre, le richérisme ", Revue d'histoire moderne, 1930, p. 257-261 et 329334.

66. Sur le gouvernement aristocratique conjoint des prêtres et des évêques dans l'Église selon Richer, voir Ph. Denis, «Edmond Richer, protestant malgré lui? », art. cit., p. 352. 
22 avril 1605. Par un relief d'appel comme d'abus, l'avocat parisien adressa alors une seconde requête au Grand Conseil, en stigmatisant la dénaturation de ses propos par Rose. Réfutant toute intention de nuire à la hiérarchie ecclésiastique, « les evesques ont été créés pour tenir l'Eglise en unité et éviter le schisme », Leschassier précise que la proposition accordant l'ordination commune des prêtres par les prêtres et les évêques n'est valide que si l'on souligne la collégialité des prêtres : « Mais il y aurait erreur de calcul qui argumenteroit $a$ presbytero ad presbyterium. Car le prestre simple est une unité et le presbyterium est la multitude et la compagnie d'un Sénat qui ne peut estre sans l'evesque qui est son chef $\rangle^{67}$. Osant convoquer l'autorité des décrets tridentins ${ }^{68}$ ou encore celle des réformes liturgiques de Clément VIII, Leschassier maintient et amplifie l'idée d'une supériorité des conciles anciens comme source de l'ecclésiologie gallicane : «Et toutesfois un prestre n'estoit point si incapable de presider aux ordres, qu'il ne le pût au nom de l'evesque, et par sa delegation, ainsi qu'il se voit dans le code canonique de l'Eglise primitive, confirmé par Justinian, et encore dans le corpus canonum ancien de Rome, par le douziesme canon du concile d'Ancyre qui est dans l'un et l'autre code $»^{69}$.

Que la censure ecclésiastique ait « changé le nom et les paroles du suppliant» fait dire à Alain Viala que Leschassier fut sans doute un pionnier dans l'histoire de la reconnaissance de la propriété intellectuelle et littéraire ${ }^{70}$. Mais le plus grave était qu'une juridiction ecclésiastique ait osé déjuger une cour souveraine et le chancelier qui avaient déjà examiné les écrits de Leschassier. Cet empiètement clérical pouvait être perçu comme la menace «d'une infame et hideuse inquisition $»^{71}$. Du reste, les syndics du clergé, conscients des répercussions politiques de l'appel comme d'abus

67. Discours sur ledit relief d'appel comme d'abus in Les Euvres de M. Jacques Leschassier..., p. 326.

68. Ibid., p. 325 « Le concile de Trente veut que les chapitres des eglises cathédrales, qui sont comme dit le pape partie d'un même corps, dont l'evesque est le chef, assistent aux ordres avec luy pour conferer avec luy par l'imposition de leurs mains cette amplitude de grace, sans laquelle selon saint Thomas ceux qui prennent l'ordre de pretrise ne pourroient rien faire de leur ordre ou fonction ».

69. Ibid., p. 325.

70. Alain Viala, Naissance de l'écrivain, Paris, 1985, p. 87-88.

71. Les syndics du clergé craingnans qu'à l'occasion de l'appel comme d'abus interjeté par Jacques Leschassier..., acte du 22 décembre 1607, p. 2 : Bibliothèque Sainte-Geneviève, $8^{\mathrm{E}} 3333$ inv. 1944 FA (P. 7 bis). 
interjeté par Leschassier, prirent les devants pour désamorcer la colère prévisible des officiers royaux. Ils désavouèrent publiquement la censure du 22 avril 1605 en faisant savoir au chancelier, et à Nicolas Hennequin qui présidait le Grand Conseil, que cette censure faute d'avoir été obtenue en assemblée plénière n'avait aucune légitimité ${ }^{72}$. En outre, ils reconnaissaient en Rose «le calomnieur et forgeron » du libelle contenant la censure et «l'opprobre que cet imposteur faisoit à deux ordres de ce royaume, le clergé et la justice $\gg^{73}$.

Du côté du pouvoir royal, l'occasion s'offrait pour Henri IV de démontrer qu'il était le défenseur des libertés gallicanes à la manière des empereurs chrétiens ou de Charlemagne qui les défendirent des empiètements pontificaux ${ }^{74}$. Mais le roi chercha d'abord à se montrer pacificateur et conciliant : il demanda au chancelier Bellièvre de constituer une commission de trois ou quatre conseillers du Conseil privé pour tenter de mettre fin au conflit « affin que lesdicts evesque et chanoines vivent mieulx ensemble et en repos qu'ils n'on faict et ne font : et cela tournera à l'honneur et gloire de Dieu $\gg^{75}$.

L'évêque fut finalement sommé de se désavouer et, après avoir refusé de se présenter devant le Grand Conseil, fut condamné par contumace (22 décembre 1607); la censure de l'assemblée du clergé fut « déclarée nulle et abusive avec injonction de la biffer et de la rayer dans les registres où elle avoit été insérée $»^{76}$. Le triomphe de

72. Sur Nicolas Hennequin, ancien ligueur, maître des requêtes et président du Grand Conseil depuis 1588, voir Camille Trani, « Les officiers du Grand Conseil », Mémoires de la fédération des sociétés historiques et archéologiques de Paris et de l'île-de-France, t. 42, 1991, p. 153.

73. Les syndics du clergé..., p. 1.

74. Joseph Lecler, «Qu'est-ce que les libertés de l’Église gallicane?», Recherches de science religieuse, XxIv, 1934, p. 72, précise que Leschassier lui-même considérait que «le roi à l'autorité de Josias ou de Constantin » pour défendre les libertés gallicanes.

75. Jules Berger de Xivrey, Recueil des lettres missives de Henri IV, t. VII, Paris, 1858, p. 206 : lettre-missive du 30 avril 1607. Avis du Roi Henri IV, au conseil d'Etat, prescrivant la nomination d'un prud'homme dans la cause d'entre l'évêque et les chanoines de Senlis, acte royal du 16 avril 1608 : BnF E-2458.

76. Les Euvres de M. Jacques Leschassier..., p. 378-380. Arrêt du Grand Conseil du 22 décembre 1607, statuant sur l'appel comme d'abus interjeté par Jacques Leschassier, avocat au parlement, d'un jugement rendu par l'assemblée générale du clergé entre l'evesque de Senlis, Antoine Roze, et son chapitre, le 22 août 1606 : Bnf 8-FM-3135. 
Leschassier, « grand prêtre de la religion royale » pour reprendre la formule de Joël Cornette ${ }^{77}$, était complet.

\section{... en résonance avec d'autres conflits}

L'affaire des chanoines de Senlis n'est pas un cas isolé. Elle rejoint notamment celle du bréviaire des chanoines d'Angers évoquée devant le Parlement de Paris. En 1603, Louis Servin, avocat général du Parlement, publie en effet un plaidoyer en faveur du chapitre collégial Sainte-Trinité d'Angers opposé à l'évêque Charles Miron. Ce dernier, en bon prélat tridentin, souhaitait remplacer le bréviaire local par un bréviaire romain. Servin, en défenseur lui aussi de l'Église gallicane, fondait son argumentation sur l'autorité d'un rescrit de la Sorbonne (1583).

Le procès, opposant les érudits gallicans du Parlement défenseurs des particularismes des Églises locales au haut clergé tridentin sinon ultramontain désireux d'unification liturgique, fit grand bruit dans la capitale. Un premier jugement de la cour souveraine obligea l'évêque d'Angers à se conformer aux usages liturgiques locaux (27 février 1603). Inquiet du développement et de la publicité de cette affaire, le nonce du pape Innocenzo del Bufalo tenta en vain de convaincre le chancelier Bellièvre d'interdire la publication de l'arrêt et du rescrit.

L'affaire s'envenima ensuite, non sans analogies avec celle des chanoines de Senlis : sans doute sollicitée par Miron, une assemblée de la Sorbonne examina le rescrit et nia son authenticité le 16 février 1604. La faculté de théologie alla jusqu'à condamner Servin comme schismatique et hérétique après avoir tenté en vain de faire censurer son livre ${ }^{78}$. Furieux de cette initiative, le premier président du Parlement de Paris menaça de poursuivre les théologiens pour crime de lèse-majesté. En obtenant l'évocation de l'affaire devant le chancelier, le nonce parvint à calmer la situa$\operatorname{tion}^{79}$. Mais comme Leschassier à Senlis, Servin avait pu mettre en

77. Joël Cornette, La monarchie entre Renaissance et Révolution (15151792), Paris, 2000, p. 156.

78. Pour l'analyse de l'affaire du bréviaire d'Angers et ses répercussions dans la nation gallicane, voir Jotham Parsons, The Church in the Republic. Gallicanism and Political Ideology in Renaissance France, Washington, 2004, p. 212-217.

79. Correspondance du nonce en France Innocenzo del Bufalo, évêque de Camerino, éd. B. Barbiche, Rome-Paris, (« Acta Nuntiaturae Gallicae» 4), 1964, p. 56. 
lumière l'idée que les prérogatives épiscopales, ici en matière liturgique, étaient bornées sinon partagées par l'autorité des collèges de chanoines ${ }^{80}$. L'affrontement presque contemporain entre l'archevêque François de Sourdis - qui de sa seule autorité tente d'imposer le bréviaire romain et d'aménager l'espace liturgique de sa cathédrale - et le chapitre cathédral de Bordeaux est du même registre ${ }^{81}$.

À l'échelle nationale, les affaires d'Angers et de Senlis, défendues par de grandes figures de la robe parisienne, renforçaient l'idée d'une Église de France, réellement émancipée de la tutelle pontificale, et enchâssée à l'intérieur d'un État royal garant des coutumes et traditions locales. Mais à l'échelle locale la portée de ces conflits s'avère sensiblement différente.

\section{LES RÉPERCUSSIONS LOCALES : LA PROMOTION D'UN CATHOLICISME ROYAL SANS ANTIROMANISME}

Certes le succès de la procédure de l'appel comme d'abus, l'arme des gallicans, avait de quoi fragiliser l'autorité épiscopale. Pourtant, les catholiques royaux de Senlis, attachés à la succession apostolique qui faisait de leur saint patron, l'évêque évangélisateur Rieul, une source de légitimité politique, ne pouvaient aller trop loin dans la critique de l'essence du pouvoir épiscopal sans risquer de porter atteinte à l'équilibre des pouvoirs urbains. Comment, dans ces circonstances, rendre compte de l'hostilité croissante dont Antoine Rose fut l'objet ${ }^{82}$

\section{Le roi plutôt que l'évêque}

Les élites dirigeantes, sans doute outrées du manque de civisme de l'évêque (rejet des commémorations de la délivrance du siège et de l'escalade, refus de l'offrande du drap d'or à la collégiale Saint-Rieul) et de son intransigeance lors de l'affaire Leschassier,

80. J. Parsons, op. cit., p. 215.

81. Ph. Loupès, Chapitres et chanoines en Guyenne aux XVII et XVIII ${ }^{e}$ siècles, Paris, Éditions de l'École des Hautes Études en Sciences Sociales, 1985, p. 360361.

82. Rose permute son siège avec le cardinal François de La Rochefoucauld le I ${ }^{\mathrm{er}}$ mars 1610, voir J. Bergin, Cardinal de La Rochefoucauld. Leadership and Reform in the French Church, New Haven et Londres, 1987. 
adoptèrent une mesure de rétorsion spectaculaire: en juillet 1607, l'assemblée de ville décida que Jacques Le Rat, marguillier de l'église Saint-Aignan, ferait « oster les armoiries de M. Antoine Roze evesque de Senlis qu'il avoit fait peindre à la monstre de l'orloge de l'eglise St Aignan et y seroient seullement laissées celles du roy et de Monseigneur le daulphin $\gg{ }^{83}$.

Pour mieux saisir la portée symbolique de cette décision, il faut préciser que l'église Saint-Aignan était l'un des grands sanctuaires civiques de la ville, le plus proche de l'hôtel de ville, là où pendant le Moyen Âge les bourgeois de la commune élirent leurs sépultures, là où une messe du Saint-Esprit était dite à chaque élection d'un nouvel échevin. La décision d'effacer les armoiries de l'évêque pour les remplacer par celles du roi et du dauphin témoigne donc d'une volonté forte d'affirmer le service exclusif du roi, plus précisément d'une nouvelle dynastie (le roi et son dauphin), comme l'identité et la raison d'être de la municipalité. Est-ce un hasard si au même moment (1608) les comptes de la ville enregistrent des dépenses nouvelles pour célébrer les anniversaires de la délivrance du siège et de « l'escalade » ${ }^{84}$ Senlis choisit donc le roi plutôt que l'évêque. Faut-il y voir une forme de sécularisation ou encore d'une déconfessionnalisation ${ }^{85}$

\section{Gallicans et dévots à la fois}

En réalité, les élites qui montrèrent tant de défiance à l'égard d'Antoine Rose n'en étaient pas moins des catholiques zélés, favorables à l'installation d'ordres nouveaux (Capucins en 1609), attachés à la piété eucharistique (confrérie du Saint Sacrement en 1618), dévoués à l'action charitable (bureau des pauvres, création d'un hôpital en $1610^{86}$ ). Autant de marqueurs confessionnels qui

83. Arch. dép. Oise, BB6, fol. $532 \mathrm{v}^{0}-533 \mathrm{r}^{\circ}$.

84. Arch. dép. Oise, CC 98, fol. $8 \mathrm{v}^{\circ}$; CC 106 fol. $25 \mathrm{v}^{\circ}$ (4 juillet 1614 et 18 mai 1615); ibid., fol. $34 \mathrm{v}^{\mathrm{o}}-35 \mathrm{r}^{\mathrm{o}}$ (1616); ibid., fol. $40 \mathrm{r}^{\mathrm{o}}$ et $54 \mathrm{v}^{\mathrm{o}}(1617)$.

85. Arlette Jouanna, «L'édit de Nantes et le processus de sécularisation de l'État», Paix des armes. Paix des âmes. Actes du colloque de Pau, dir. P. Mironneau et I. Pébay-Clottes, Paris, 2000, p. 481-486. Sur l'affirmation d'un État monarchique impartial échappant à l'emprise des confessions, voir Olivier Christin, La paix de religion. L'autonomisation de la raison politique au XVI siècle, Paris, 1997, p. 189-197.

86. Bibliothèque municipale de Senlis, coll. Afforty, t. XII, p. 1588, 25 janvier 1610 . 
les rendent proches d'un catholicisme de «Contre-Réforme ». De surcroit, l'action pastorale du cardinal de La Rochefoucauld rencontra manifestement un large succès tant auprès du clergé local que des fidèles, Senlis devenant un $"$ petit Milan $»^{87}$. À tel point que, sous l'impulsion épiscopale, le culte de saint Charles Borromée fut introduit en 1623 dans l'une des chapelles du sanctuaire de Saint-Aignan ${ }^{88}$.

Toutefois, et contrairement à son prédécesseur, La Rochefoucauld respecta scrupuleusement les rituels locaux. Ainsi, lors de son entrée solennelle, il se plia à la coutume qui visait à honorer l'évêque évangélisateur, et fit don de riches ornements liturgiques aux deux principaux établissements ecclésiastiques : la cathédrale Notre-Dame et la collégiale Saint-Rieul, siège du culte de l'évêque évangélisateur de Senlis ${ }^{89}$. De façon significative, l'évêque dédicaça en 1614 l'église des Capucins de Senlis « en l'honneur de Nostre Dame et des huict saincts evesques de Senlis $»^{90}$. La célébration liturgique rejoignait ici la volonté initiale de l'avocat Nicolas de Cornoailles qui, en fondant l'église des Capucins à Senlis, souhaitait honorer saint Rieul et la succession apostolique ${ }^{91}$. Ces éléments aident à comprendre le double échec d'Antoine Rose : avoir négligé le poids $\mathrm{du}$ corporatisme (les collèges de chanoines et la municipalité); avoir voulu prolonger une réforme tridentine sans tenir compte des traditions locales gallicanes ${ }^{92}$ (culte de saint Rieul intégré

87. J. Bergin, op. cit., p. 110-118.

88. Charles Jaulnay, Le parfait prélat ou la vie et miracles de saint Rieule second evesque d'Arles, depuis second evesque de Paris et ensuite premier evesque, apostre et patron de la ville et diocese de Senlis. Avec une histoire des choses plus remarquables arrivées depuis plus de 1500 ans en diverses parties du monde, tant du fait ecclesiastique que seculier, sous l'episcopat de chacun evesque de Senlis, au nombre de 88, Paris chez Jean Paslé, 1648, p. 633.

89. Ibid., p. 635.

90. Ibid., p. 630-631.

91. Arch. dép. Oise, annexe de Senlis BB 6, fol. $550 \mathrm{v}^{0}$ : dédicace du 27 juillet 1614. Voir aussi Bibl. mun. de Senlis, coll. Afforty, t. XI, p. 140. En 1637, un autre Nicolas de Cornoailles, official de Senlis et fils du précédent, imita la dévotion de son père : Arch. dép. Oise à Beauvais : G 2201.

92. Un autre exemple de conflit entre des notables attachés à leurs traditions et un évêque cherchant à éradiquer les «superstitions" nous est fourni à Agen où l'évêque Pierre Sauveur osa soutenir en 1622 que le saint patron local (saint Caprais) n'avait jamais été évêque. La ville réagit vigoureusement par l'érudition en faisant publier un Bref recueil de l'histoire de Saint-Caprais, voir Gregory Hanlon, L'univers des gens de bien : culture et comportements des élites urbaines en Agenais-Condomois au XVII siècle, Bordeaux, 1999, p. 278-279. 
aux commémorations civiques) qui sanctifiaient le gouvernement municipal ${ }^{93}$.

Les théories sur le code canonique de l'Église primitive de Leschassier fascinèrent durablement les grands gallicans parisiens qui, grâce à leurs recherches érudites, pensaient le particulier des situations locales pour mieux défendre l'idée générale d'une Église nationale préservée de la tutelle du Saint-Siège. La défense des chanoines de Senlis est alors emblématique d'un mouvement général qui, au sortir des guerres de Religion, voit le magistère intellectuel des grands robins se substituer à l'autorité doctrinale des théologiens de l'Université ${ }^{94}$.

En revanche, les thèses savantes de Leschassier sur les anciens conciles ne suscitèrent guère, pour autant qu'on puisse en juger, d'enthousiasme au sein des élites locales. Et il est vrai que la conception d'une législation primitive - qui aurait subi de multiples retranchements de la part de la législation pontificale - ruinait le mythe d'une continuité historique des libertés gallicanes auquel les catholiques royaux de Senlis, ardents promoteurs de la succession apostolique, étaient attachés ${ }^{95}$. Enfin il est frappant de voir à quel point ces turbulences gallicanes du début du XVII ${ }^{\mathrm{e}}$ siècle, à Senlis, Angers ou Bordeaux, imposent la figure arbitrale du roi. Henri IV devait tenir à cette image de conciliateur, qu'il adopte aussi sur le plan international en offrant sa médiation dans le conflit opposant Venise au Pape, car elle contribuait à fortifier sa stature

93. Nous rejoignons ainsi Simon Ditchfield qui montre que, contrairement à la centralisation romaine sur les dévotions locales et à la Sozialdisziplinierung que l'on prête à la Contre-Réforme, un évêque comme Charles Borromée, dans son expérience pastorale milanaise, a parfaitement tenu compte des liturgies locales et des saints du Milanais, voir Simon Ditchfield, «San Carlo and the cult of saints ", Cultura e spiritualità borromaica tra cinque et seicento, Studia borromaica. Saggi e documenti diu storia religiosa e civile della prima età moderna, $\mathrm{n}^{\circ}$ 20, éd. Franco Buzzi et Maria-Luisa Frosio, Milan, 2006, p. $145-154$.

94. Robert Descimon, «Penser librement son intolérance: le président Jacques-Auguste de Thou (1553-1617) et l'épître dédicatoire des Historiae sui temporis (1604) », La Licorne, n 61 ("La liberté de Pensée », numéro dirigé par F. Lecercle), 2002, p. 84-85.

95. Sur l'opposition des thèses de Leschassier à l'idée d'une continuité historique des libertés gallicanes, W. J. Bouwsma, «Gallicanism and the Nature of Christendom », art. cit., p. 824. 
de prince chrétien ${ }^{96}$. Le double processus politique qui semble alors à l'œuvre - attachement renforcé des sujets à la personne royale et construction d'un État monarchique situé hors du champ des passions religieuses - constitue sans doute l'une des dynamiques de la construction naissante de l'absolutisme bourbonien ${ }^{97}$.

Thierry.Amalou@wanadoo.fr

96. S. Hermann De Franceschi, « La diplomatie henricienne et les ambitions françaises de suprématie temporelle sur la république chrétienne », Histoire, économie et société, 2004, $\mathrm{n}^{\circ}$ 4, p. 551-585.

97. Dale K. Van Kley, Les origines religieuses de la Révolution française, 1560-1791, Paris, 2002, p. 61-62. 